\title{
Role of tropomyosin isoforms in the calcium sensitivity of striated muscle thin filaments
}

\author{
Sabrina E. Boussouf · Robin Maytum • \\ Kornelia Jaquet $\cdot$ Michael A. Geeves
}

Received: 5 February 2007 / Accepted: 20 March 2007/Published online: 14 April 2007

(C) Springer Science+Business Media B.V. 2007

\begin{abstract}
We have expressed $\alpha$ \& $\beta$ isoforms of mammalian striated muscle tropomyosin $(\mathrm{Tm})$ and $\alpha$-Tm carrying the D175N or E180G cardiomyopathy mutations. In each case the Tm carries an Ala-Ser N-terminal extension to mimic the acetylation of the native Tm. We show that these Ala-Ser modified proteins are good analogues of the native $\mathrm{Tm}$ in the assays used here. We go on to use an in vitro kinetic approach to define the assembly of actin filaments with the Tm isoforms with either a cardiac or a skeletal muscle troponin (cTn, skTn). With skTn the calcium sensitivity of the actin filament is the same for $\alpha \&$ $\beta$-Tm and there is little change with the mutant Tms. For cTn switching from $\alpha$ to $\beta$-Tm causes an increase of calcium sensitivity of $0.2 \mathrm{pCa}$ units. D175N is very similar to the wild type $\alpha$-Tm and E180G shows a small increase in calcium sensitivity of about $0.1 \mathrm{pCa}$ unit. The formation of the switched-off blocked-state of the actin filament is independent of the Tm isoform but does differ for cardiac versus skeletal $\mathrm{Tn}$. The in vitro assays developed here
\end{abstract}

S. E. Boussouf · R. Maytum · M. A. Geeves ( $\square)$

Department of Biosciences, University of Kent, Canterbury CT2

7NJ, UK

e-mail: m.a.geeves@kent.ac.uk

K. Jaquet

St Josef-Hospital/Bergmannsheil, Clinic of the Ruhr-University of Bochum, 44791 Bochum, Germany

Present Address:

S. E. Boussouf

National Institute of Medical Research, The Ridgeway, Mill Hill, London, UK

Present Address:

R. Maytum

Department of Biosciences, Queen Mary \& Westfield College,

University of London, London, UK provide a novel, simple and efficient method for assaying the behaviour of expressed thin filament proteins.

Keywords Actin · Ca-regulation - Skeletal muscle . Cardiac muscle $\cdot$ Cardiomyopathies
Abbreviations
Tm Tropomyosin
$\alpha-\mathrm{Tm} \quad$ Alpha tropomyosin
$\beta$-Tm Beta tropomyosin
A Actin
S1 Myosin subfragment 1
skTn Skeletal muscle troponin
cTn Cardiac muscle troponin
PKA Protein kinase A
FHC Familial Hypertrophic Cardiomyopathies

\section{Introduction}

Striated muscle contraction is activated by calcium binding to the thin filament protein troponin (Tn; Ebashi et al. 1971; Gordon et al. 2000). The activation signal is coupled to the movement of tropomyosin $(\mathrm{Tm})$ over the surface of the actin monomers of the thin filament (Craig and Lehman 2001; Pirani et al. 2006; Tao 1990). This movement relieves the inhibition of myosin binding and allows the ATP driven actomyosin contractile cycle to operate. The exact details of the activation process are still the subject of active research but most observers agree that a calcium induced change in the conformation of Tn (Takeda et al. 2003; Vinogradova et al. 2005) is coupled to changes in the interaction between $\mathrm{Tm}$ and actin, and this leads to 
activation of contraction. Here we are concerned with the role of the different isoforms of striated muscle Tm, $\alpha$ and $\beta$ and how these interact with skeletal or cardiac Tn (skTn, cTn) to influence the calcium sensitivity of contraction.

Striated muscle $\mathrm{Tm}$ is $\alpha$-helical rod-like protein that forms a parallel coiled-coil dimer which polymerises headto-tail along the surface of actin. Mammalian striated muscle cells, both cardiac and skeletal, express Tm from two genes (TM1 \& TM2) and at the protein level these are known as $\alpha$-Tm \& $\beta$-Tm (Lees-Miller and Helfman 1991; Helfman 1994). The $\alpha$-Tm is more stable and the $\alpha \alpha$ dimer predominates in most muscles. $\beta$-Tm is relatively unstable as a $\beta \beta$ dimer and is rarely found but the $\alpha \beta$ heterodimer is stable and most mammalian striated muscle contains both $\alpha \alpha \& \alpha \beta$-Tm (Lehrer and Joseph 1987; Lehrer et al. 1989; Lehrer and Qian 1990; Jagatheesan et al. 2003). The physiological role of $\alpha \beta$ vs. $\alpha \alpha$-Tm is not understood but it is known that the proportion of $\alpha \beta$ is increased in the slower contracting muscles of larger animals and can increase under certain diseased conditions (Heeley et al. 1983; Perry 2001). The $\alpha$-Tm and $\beta$-Tm isoforms are identical in skeletal and cardiac muscle, the only difference is the proportion of $\alpha \alpha$ vs. $\alpha \beta$ can vary. Cardiac muscles of small mammals express virtually pure $\alpha \alpha-\mathrm{Tm}$ and transgenic mouse lines over-expressing $\beta$-Tm showed changes in calcium sensitivity and cardiac abnormalities that were lethal (Muthuchamy et al. 1995, 1998). To date there has been no detailed study of the calcium regulation of actin filaments containing $\beta$-Tm compared to $\alpha$-Tm in vitro.

Alpha and beta Tm isoforms are found in all vertebrates. The sequence alignment of rat $\alpha \& \beta$-Tm is shown in Fig. 1 . The sequence is $95 \%$ conserved with $87 \%$ identity. The 36 alterations in amino acid residues are distributed throughout the sequence but are more predominant in the C-terminal half. Individually both $\alpha$-Tm and $\beta$-Tm sequences are highly conserved. Rat and rabbit $\beta$-Tm are identical and differ from human $\beta$-Tm at a single site, D66 in rat is E66 in human (swiss-prot P58775, P07951 \& P58776 for rat human and rabbit $\beta$-Tm respectively). Rat $\alpha$-Tm differs from rabbit at one site, K279 is N279 in rabbit, human has the same N279 as rabbit and K220 of rat and rabbit is replaced by R220 in human Tm (swiss-prot P04692, P09493, P58772).

We are interested in the functional significance of the $\mathrm{Tm}$ isoforms and have expressed the two rat $\mathrm{Tm}$ isoforms in E. coli with the Ala-Ser N-terminal extension which is used to mimic the native N-terminal acetylation (Monteiro et al. 1994). We show here that this appears to be a good model for the native $\mathrm{Tm}$ in the assays used. We have also made the non-conservative, K279N, mutation in $\alpha$-Tm to make the rat sequence identical to the rabbit and only the one, K220R, conservative difference to the human sequence. Currently it is not possible to reliably assemble
$100 \% \alpha \beta$-Tm and therefore as a first step we examine the properties of the $\alpha \alpha-\& \beta \beta$-Tm isoforms and compare then to the well characterised native rabbit $\mathrm{Tm}$ which is an approximately 50:50 mixute of $\alpha \alpha$ - and $\alpha \beta$-Tm in skeletal muscle.

In this study we assemble thin filaments with $\alpha \alpha$-Tm, $\beta \beta-\mathrm{Tm}$, then compare the calcium sensitivity of the thin filaments when assembled with rabbit skTn or human cTn and show that skeletal and cardiac Tns have a different sensitivity to the Tm isoforms. Two well-characterised point mutations of $\alpha \alpha-\mathrm{Tm}$ are also studied, E180G or D175N, using the same approach. (Thierfelder et al. 1994; Golitsina et al. 1997; Kremneva et al. 2004). These mutations are associated with Familial Hypertrophic Cardiomyopathies (FHC) but no phenotype has been reported with the expression of the mutation in skeletal muscle. We show that the $\alpha \alpha-T m$ carrying the mutations decrease the calcium required to activate the filament and the effect is smaller with skTn than cTn. However, the effects are small in each case (0.03-0.13 pCa unit).

The in vitro assays of calcium sensitivity used here are ones that we developed previously for defining the calcium sensitivity of skeletal and cardiac Tn (McKillop and Geeves 1993; Head et al. 1995; Reiffert et al. 1996). This measures the calcium sensitivity of the rate constant of myosin subfragment 1 (S1) binding to a large excess of thin filaments. An advantage of this assay is that it only measures the accessibility of the actin sites to the myosin heads. It does not measure the process of switching on of the filament; the assay is independent to a significant extent of the model used to explain activation of the filament. The method used here has not been adopted more widely for in vitro assays of calcium regulation. We demonstrate here that it can be used for a wide range of different thin filaments and will complement studies at the myofibril or muscle fibre level.

\section{Materials and methods}

\section{Protein preparations}

Rabbit actin was prepared as described by Spudich and Watt (1971) and its concentration was determined using the $280 \mathrm{~nm}$ extinction coefficient of $1.104 \mathrm{mg}^{-1} \mathrm{ml} \mathrm{cm}^{-1}$ and a molecular mass of 42,000 Da. The procedure for the pyrene labelling of actin at Cys-374 was described by Criddle et al. (1985).

Rabbit myosin subfragment (S1) was prepared as described by Weeds and Taylor (1975). Its concentration was calculated using absorbance measurements at $280 \mathrm{~nm}$ with an extinction coefficient of $0.79 \mathrm{mg}^{-1} \mathrm{ml} \mathrm{cm}^{-1}$ and a molecular mass of 115,000 Da. 


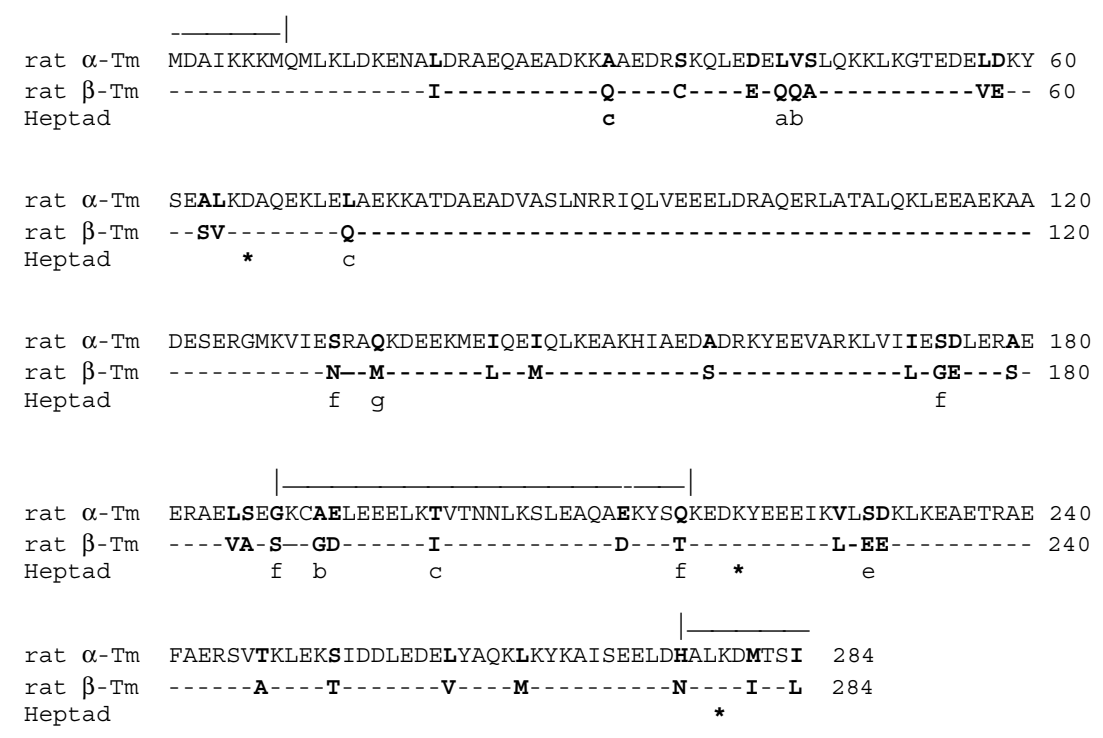

Fig. 1 Sequence alignment of rat $\alpha$ and $\beta \mathrm{Tm}$. Sequence identity is indicated by a dash (-). The position in the heptad repeat is shown for the 12 non-conservative sequence changes $(\mathrm{a}-\mathrm{g})$. The stars at position $66(\beta-\mathrm{Tm}, \mathrm{D}-\mathrm{E}), 220(\alpha-\mathrm{Tm} \mathrm{K}-\mathrm{R})$ and $279(\alpha-\mathrm{Tm} \mathrm{K}-\mathrm{N})$ mark the sites where differences are found between rat and human Tm. Note that in the rat $\alpha$-Tm used here we have made the K279N mutation to

Recombinant rat $\beta$-Tm with the Ala-Ser extension was amplified from a CGN vector (gift from Dr D.Helfman) using PCR primers which allowed the cloning of the entire coding sequence and the introduction of NdeI and Bam $\mathbf{H I}$ restriction sites. The gene was then introduced into pJC20 vector. The Ala-Ser extension shown to mimic acetylation (Monteiro et al. 1994) was introduced at the N-terminal end of the sequence using a $5^{\prime}$ primer with the additional Met-Ala.Ser amino acid sequences. The forward and reverse primer sequences were

5'-GGAATTCCATATGGCGAGCATGGACGCCATCAAGAAGAAGATGC-3'

5'-CGCGGATCCTCAGAGGGAAGTGATGTCATTGAGCG-3'

Recombinant rat $\alpha$-Tm WT (with the point mutation $\mathrm{K} 279 \mathrm{~N}$ as in human $\alpha$-Tm) and $\alpha$-Tm containing FHC mutants D175N and E180G expressed with the Ala-Ser extension were cloned as described in Kremneva et al. (2004).

All recombinant $\mathrm{Tm}$ were over-expressed in BL-21 DE3(pLys) host cells and purified as described in Maytum et al. (2000) and Kremneva et al. (2004). Yields of pure protein were typically $20-40 \mathrm{mg}$ from a 21 culture. In each case the purified protein was characterized by mass spectrometry and the molecular mass agreed with that predicted from the sequence to within $3 \mathrm{Da}$. Protein concentrations were determined using absorbance at $280 \mathrm{~nm}$ with an extinction coefficient of $0.276 \mathrm{mg}^{-1} \mathrm{ml} \mathrm{cm}^{-1}$ and monomer molecular mass of 32,800 Da. make the construct identical to rabbit. Also this construct differs only by one amino acid from human $\alpha$-Tm. The line above the sequences marks the potential Tn interaction sites, around exon 6 (residues 188-217) and the 8-9 residue Tm-Tm overlap region at the $\mathrm{N}$ and C-termini thought to be a site of TnT1 binding

Rabbit skTnTm complex from which skTn was isolated was obtained using the methods of Ebashi et al. (1971) and Greaser and Gergely (1971).

Human cardiac Tn was expressed, reconstituted and phosphorylated with the catalytic subunit of protein kinase A (PKA) as described by Deng et al. (2001). This resulted in the bisphosphorylation of the TnI subunit on Ser 22 and 23. The level of phosphorylation was checked by isoelectric focusing (Reiffert et al. 1999).

Thin filaments were assembled by incubating actin with the required concentrations of $\mathrm{Tm}$ and $\mathrm{Tn}$ (standard ratio $\mathrm{A}: \operatorname{Tm}: \operatorname{Tn}$ of $7: 2: 2$ ) for $1 \mathrm{~h}$ before using in the measurements.

\section{Co-sedimentation and densitometry}

Co-sedimentation assays were performed at $20^{\circ} \mathrm{C}$ by mixing $10 \mu \mathrm{M}$ polymerized F-actin with $0-3 \mu \mathrm{M} \mathrm{Tm}$ in $20 \mathrm{mM}$ MOPS, $100 \mathrm{mM} \mathrm{KCl}, 2 \mathrm{mM} \mathrm{MgCl}_{2}$, pH 7.0 to reach a total volume of $100 \mu \mathrm{l}$. The samples were then incubated for at least $1 \mathrm{~h}$. The polymerized actin and the $\mathrm{Tm}$ bound to it could then be pelleted by centrifugation at $100,000 \mathrm{~g}$ for $20 \mathrm{~min}$ (Beckman TL100A). Equivalent samples of pellet and supernatant were then run on a $12 \%$ SDS-PAGE gels (Laemmli 1970), which were then stained with Coomassie Blue G-250. Densitometry analyses of the protein bands were carried out using an Epson 1640SU scanner and the Image-PC program (Scion Corp. based upon NIH-Image). The density of protein bands was 
measured by comparison to an integrated Kodak neutral density step tablet.

Calcium sensitivity measurements using stopped-flow technique

The calcium-dependent rate of S1 binding to pyrene-labelled actin was measured by monitoring the change in pyrene fluorescence in the stopped-flow machine (SF-61DX2 spectrophotometer, Hi-Tech Scientific). All the measurements were carried out at $20^{\circ} \mathrm{C}$. Pyrene was excited at $365 \mathrm{~nm}$ and the emitted fluorescence was detected using a KV $389 \mathrm{~nm}$ cut off filter. The obtained data was visualised and analysed using the Kinetasyst software provided with the instrument. The protein concentrations cited in the text are those obtained after mixing. Each transient shown is the average of at least three shots. The $k_{\text {obs }}$ values, obtained from fitting the transients to a single exponential, were plotted as a function of $\mathrm{pCa}$. The sigmoid curve was fitted to a version of the Hill equation

$k_{\text {obs }}^{\prime}=\left[\mathrm{Ca}^{2+}\right]^{h} /\left(\mathrm{K}^{h}+\left[\mathrm{Ca}^{2+}\right]^{h}\right)$

where $K$ is the calcium concentration at the mid point of the titration, $h$ is the Hill coefficient and the relative value of $k_{\text {obs }}$ is defined as the $k_{\text {obs }}^{\prime}=\left(k_{\text {obs }}-k_{8.9}\right) /\left(k_{4.5}-k_{8.9}\right)$ where the subscripts 8.9 and 4.5 refer to $k_{\text {obs }}$ values at the minimum and maximum of the the $\mathrm{pCa}$ range. The $\mathrm{pCa}$ values used were generated using mixture of $2 \mathrm{mM}$ EGTA and $2 \mathrm{mM}$ Ca-EGTA to produce the free calcium concentration required based on the affinity constants (Harrison and Bers 1987).

\section{Results}

The affinities of the expressed Tms for actin were measured in a sedimentation assay (see methods). Figure 2A shows the SDS-PAGE gels for the supernatant and pellets of a high-speed centrifugation of $10 \mu \mathrm{M}$ actin with $\alpha$-Tm in the range of $0.2-3 \mu \mathrm{M}$. The gel was subjected to a densitometer analysis and the band densities converted into concentrations of actin-bound and free Tm. The results are shown in Fig. 2 B for both AS. $\alpha$ and AS. $\beta$-Tm. The data show that the amount of Tm that sedimented with actin increased up to a maximum, which was close to the expected 1 Tm per 7 actins. The binding curves were fitted to a Hill equation and both the affinity values $\left(K_{50 \%}\right)$ and the Hill coefficient $\left(h_{A}\right)$ are shown in Table $1 . \alpha$ - and $\beta \mathrm{Tm}$ have similar affinities to actin of about $0.2 \mu \mathrm{M}$. In the absence of the AS N-terminal extension no significant binding of either Tm to actin was detected at up to $2 \mu \mathrm{M}$ concentrations. This is consistent with previously

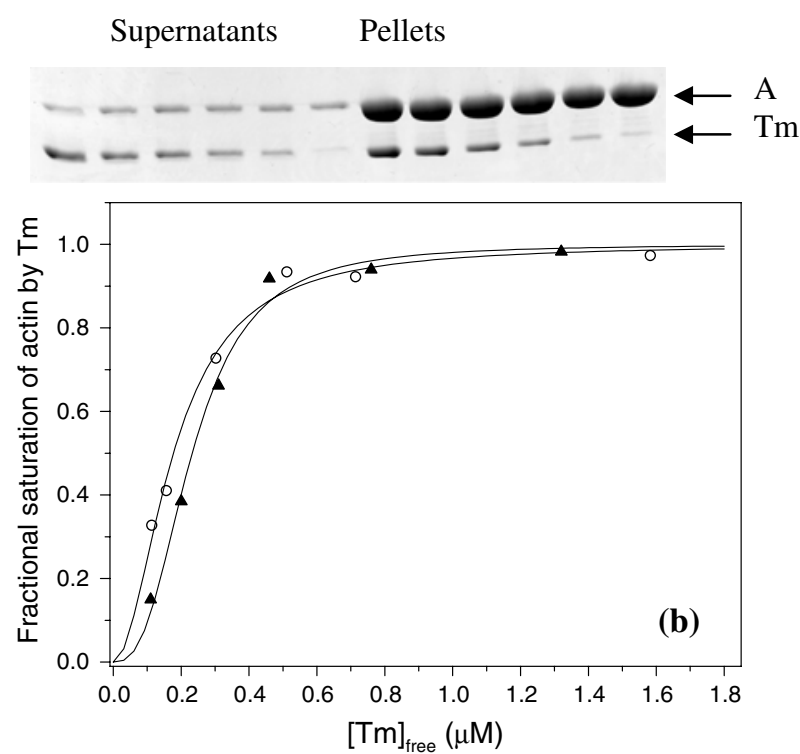

Fig. 2 Tm affinity for actin determined by co-sedimentation analysis. (A) SDS-PAGE analysis of actin and Tm co-sedimentation. Actin $(10 \mu \mathrm{M})$ was incubated with $0.2,0.5,1,1.5,2 \& 3 \mu \mathrm{M} \alpha$-Tm before the actin was sedimented. The gels show the pellets and supernatants of each sample. (B) A plot of the fractional saturation of actin by Tm as a function of free Tm concentration for AS. $\alpha-\operatorname{Tm}(O)$ and for AS. $\beta-\operatorname{Tm}(\Delta)$. The data was fitted to the Hill equation and the best-fit curves are superimposed. The best fit values for $K_{50 \%}$ (Tm concentration at which half-saturation is achieved) and the Hill coefficient $\left(h_{A}\right)$ are presented in Table 1 . Buffer conditions: $20 \mathrm{mM}$ MOPS, $100 \mathrm{mM} \mathrm{KCl}, 5 \mathrm{mM} \mathrm{MgCl} 2 \mathrm{pH} 7.0$

published data using bacterially expressed $\alpha$-Tm (Monteiro et al. 1994) and shows that the AS extension is essential for both $\alpha$ and $\beta$-Tm to bind to actin.

Table 1 also shows the affinities of actin for AS. $\alpha$-Tm with FHC mutations D175N and E180G, which we previously reported (Kremneva et al. 2004). Both FHC mutations reduce the affinity of Tm binding to actin with a greater effect in the case of E180G mutation. The Hill coefficients suggests that the weaker the affinity the more cooperative the binding of Tm to actin. The precision of the assay for affinities below $0.5 \mu \mathrm{M}$ is not high so this observed increase in Hill coefficient may be a limitation of the assay at these affinities tighter than $0.5 \mu \mathrm{M}$.

The rate at which $\mathrm{S} 1$ binds to actin is relatively independent of the presence of $\mathrm{Tm}$ and when calcium is present, addition of skTn has little effect on the observed rate constant of S1 binding (McKillop and Geeves 1993; Head et al. 1995; Schaertl et al. 1995). This is consistent with the actin.Tm and actin.TmTn (+calcium) filaments being largely in the same form as the pure actin filaments. In the absence of calcium, pre-incubation of actin with TmTn reduces the rate of $\mathrm{S} 1$ binding to actin; the form of the inhibition depends upon how the assay is performed. Figure $3 \mathrm{~A}$ shows the pyrene actin fluorescence transient when $0.25 \mu \mathrm{M} \mathrm{S} 1$ is mixed with a ten-fold excess of 
Table 1 The affinity of Tm for actin and the affinity of calcium for actin $\operatorname{Tm} T n$ thin filaments

\begin{tabular}{llcllll}
\hline & & Native $\mathrm{Tm}^{\mathrm{a}}$ & AS. $\beta$-Tm & AS. $\alpha$-Tm & AS.D175N $\alpha$-Tm & AS.E180G $\alpha$-Tm \\
\hline Tm binding to actin & $K_{50 \%}(\mu \mathrm{M})$ & $0.18 \pm 0.04$ & $0.23 \pm 0.01$ & $0.18 \pm .01$ & $0.51 \pm 0.02^{\mathrm{b}}$ & $0.68 \pm 0.01^{\mathrm{b}}$ \\
& $h_{A}$ & $1.7 \pm 0.5$ & $2.70 \pm 0.38$ & $1.95 \pm 0.17$ & $2.90 \pm 0.18^{\mathrm{b}}$ & $3.95 \pm 0.20^{\mathrm{b}}$ \\
pCa measurements with cTn & $\mathrm{pCa}_{50 \%}$ & $5.68 \pm 0.03$ & $5.80 \pm 0.07$ & $5.57 \pm 0.08$ & $5.66 \pm 0.07$ & $5.70 \pm 0.03$ \\
& $h_{C a}$ & $1.25 \pm 0.05$ & $1.20 \pm 0.10$ & $1.15 \pm 0.09$ & $1.16 \pm 0.04$ & $1.16 \pm 0.04$ \\
pCa measurements with skTn & $\mathrm{pCa}_{50 \%}$ & $6.02 \pm 0.02$ & $6.06 \pm 0.01$ & $6.05 \pm 0.03$ & $6.08 \pm 0.04$ & $6.15 \pm 0.07$ \\
& $h_{C a}$ & $1.5 \pm 0.07$ & $1.68 \pm 0.08$ & $1.50 \pm 0.07$ & $1.22 \pm 0.10$ & $1.19 \pm 0.07$ \\
\hline
\end{tabular}

Errors represent the standard error from the fit to the data

a Two types of native Tm were used. For the actin affinity measurement $\alpha \alpha-\mathrm{Tm}$ was purified from rabbit skeletal muscle. For the pCa measurements rabbit muscle Tm was used without separating the $\alpha \alpha$ and $\alpha \beta$ isoforms. cTn was phosphorylated by PKA

b Data from (Kremneva et al. 2004)

$K_{50 \%}$ is the apparent affinity of Tm for actin (free Tm concentration at 50\% saturation of actin), and $h_{A}$ is the Hill coefficient, both derived for the fit of the Hill Eq. to data of Fig. 2. $\mathrm{pCa}_{50 \%}$ and $h_{\mathrm{Ca}}$ are the equivalent values for calcium affinity and Hill coefficients for calcium switching of the thin filaments

pyr-actin in the absence or presence of a saturating level of native, rabbit-skeletal TmTn at low calcium concentrations (pCa 8.9). In the absence of TmTn the data is well described by a single exponential with $k_{\text {obs }}=5.0 \mathrm{~s}^{-1}$. Addition of TmTn sufficient to saturate the actin filament gives a similar amplitude of the transient and can also be described by a single exponential with $k_{\mathrm{obs}}=1.1 \mathrm{~s}^{-1}$, an almost 5 fold reduction in $k_{\text {obs }}$. The reduction in $k_{\text {obs }}$ by $\mathrm{TmTn}$ in the absence of calcium is consistent with previous measurements (McKillop and Geeves 1993; Head et al. 1995; Schaertl et al. 1995). The ratio of the $k_{\mathrm{obs}}$ values for TmTn with and without calcium was on average 3.5:1 also consistent with the same literature.

Figure 3B shows the reduction in $k_{\mathrm{obs}}$ as a function of TmTn concentration. A similar titration in the presence of calcium shows little effect of TmTn on $k_{\text {obs. }}$. In the absence of calcium the $k_{\mathrm{obs}}$ is a linear function of TmTn concentration up to a break point and is then independent of $\mathrm{TmTn}$ concentration. The break point occurs at $0.6 \mu \mathrm{M} \mathrm{TmTn}$. If the proteins were all $100 \%$ active and the TmTn binds with very high affinity to actin the break point would be expected to occur at $0.71 \mu \mathrm{M}$ to give an $\operatorname{actin}_{7} \operatorname{TmTn}$ complex. The titration therefore provides a way of defining the quality of the proteins and the maximum extent of inhibition of the rate of S1 binding. The consistency of the result provides a simple test of the quality of assembly of the thin filament. Assembly of thin filaments with pyr-actin and tissue purified rabbit skeletal TmTn is very consistent in our hands as is the assembly using all of our recombinant Tms with skTn. Addition of skTmTn in the presence of calcium gives no more than $15 \%$ reduction in $k_{\mathrm{obs}}$ and removing calcium reduces $k_{\text {obs }}$ further by a factor of $3.5 \pm 0.5$. Values outside this range are considered suspect and may indicate damaged troponin.

If $\mathrm{S} 1$ binding to actin at these low protein concentrations can be described as a simple single-step, bimolecular reaction then the $k_{\mathrm{obs}}$ value is defined for a pure actin filament as $k_{\mathrm{obs}}=[\mathrm{A}] \cdot k$ when actin is in excess of $\mathrm{S} 1$ and $k$ is the apparent second order rate constant for S1 binding to actin. A reduction in $k_{\mathrm{obs}}$ can be interpreted as a reduction in either the effective actin concentration or in $k$. In the presence of calcium the addition of TmTn leads to a small reduction in $k_{\mathrm{obs}}$ and reflects either a small effect of TmTn upon $k$ or an indication of the presence of some $\mathrm{TmTn}$, which cannot be activated by calcium. The removal of calcium then leads to a further 3.5 fold reduction in $k_{\text {obs }}$ and has been interpreted as due to a fraction of the actin sites being unavailable. This idea was incorporated in to the 3-state thin filament model of (McKillop and Geeves 1993). In this model TmTn in the absence of calcium is in an equilibrium between 3-states: the $\mathrm{M}$ or open and $\mathrm{C}$ or closed states to which $\mathrm{S} 1$ can bind rapidly as for pure actin filaments and the B or blocked state to which S1 cannot effectively bind. For actin $\mathrm{TmTn}$ filaments in the absence of calcium then $k_{\mathrm{obs}}=[\mathrm{A}] \cdot k K_{B} /\left(1+K_{B}\right)$ where $K_{B}$ is the equilibrium constant that defines the ratio of actin.TmTn complexes in the blocked and closed conformations $\left(\mathrm{K}_{\mathrm{B}}=[\right.$ closed $] /[$ blocked $\left.]\right) \quad$ Thus $\quad K_{B} /\left(1+K_{B}\right)=1 / 3.5$, hence $K_{B}=0.4$. This assumes the occupancy of the open conformation is negligible.

It is important to emphasise the large reduction in $k_{\mathrm{obs}}$ is the experimental observation and this is independent of the model used to interpret the data. There are other models of thin filament regulation(Tobacman and Butters 2000; Chen et al. 2001; Heeley et al. 2006) and currently there is no simple way to distinguish between these models. However, the data can be used to define aspects of the thin filament regulation that are independent of the detailed model used.

Assembly of thin filaments using cardiac Tn is more variable as reported previously (Lohmann et al. 2001) and the assay described in Fig. 3B provides a simple way to test that satisfactory thin filaments are being produced. For $\mathrm{cTn}$ 

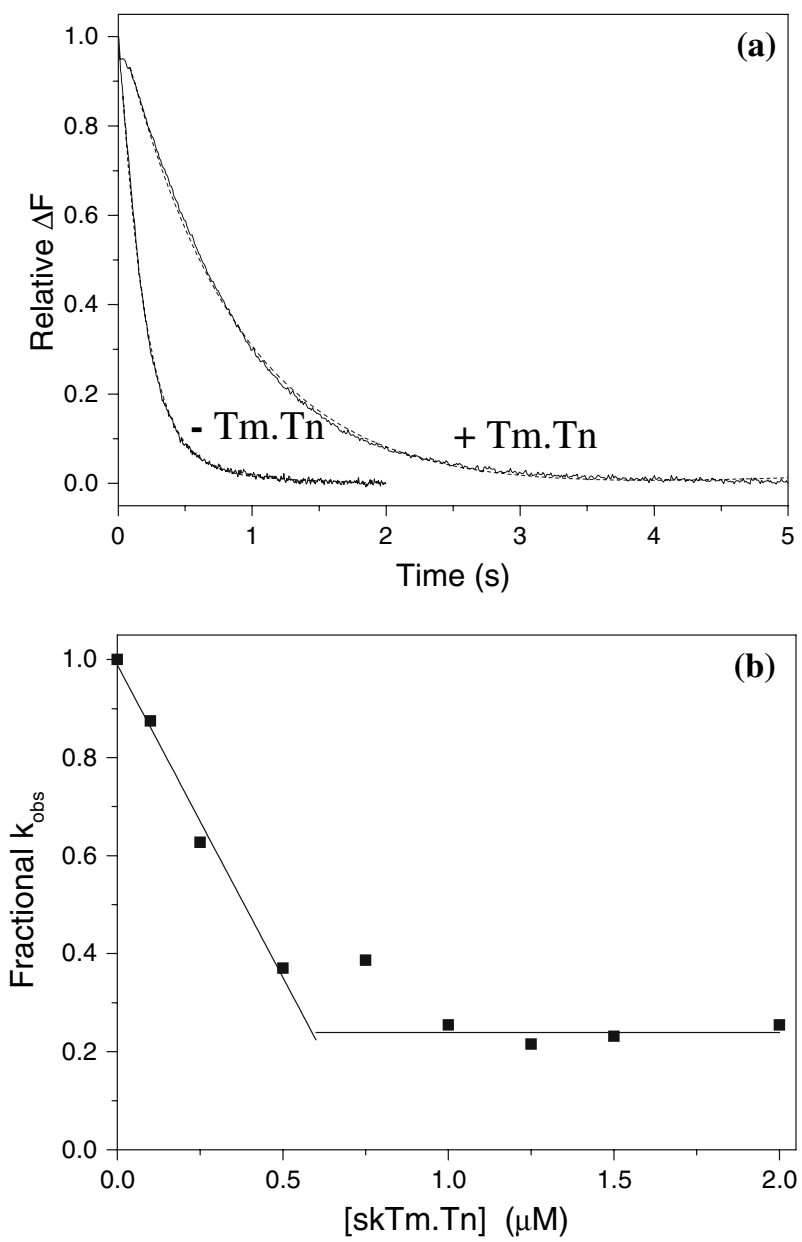

Fig. 3 Dependence of the observed rate of S1 binding to pyrene-actin in the absence of calcium on Tm.Tn concentration. (A) Observed transient when mixing $0.25 \mu \mathrm{M}$ S1 with $2.5 \mu \mathrm{M}$ pyr-actin in the presence and absence of $1 \mu \mathrm{M}$ skTmTn. The best fit singleexponential fit is superimposed with $k_{\mathrm{obs}}=5 \mathrm{~s}^{-1} \quad(-\mathrm{TmTn})$ and $k_{\text {obs }}=1.1 \mathrm{~s}^{-1}$ (+skTm.Tn). (B) The $k_{\text {obs }}$ values plotted as a function of the concentration of skTm.Tn. Data points $0-0.5 \mu \mathrm{M}$ and $1-2 \mu \mathrm{M}$ were fitted to a straight lines which are superimposed. Experimental conditions as for Fig. 2 plus 2 mM EGTA

and native $\mathrm{Tm}$ in the absence of calcium a four-fold reduction in $k_{\text {obs }}$ compared to unregulated actin is considered acceptable with saturation at between 1:7 and 2:7 ratio of $\mathrm{Tn}$ to actin. Preparations with properties outside this range were considered unacceptable and were rejected. The ratio of $k_{\mathrm{obs}}$ in the presence and absence of calcium is normally lower than for skeletal Tn with $2.6 \pm 0.3$ being typical (Maytum et al. 2003).

Similar measurements were made with each of the recombinant Tms using an actin:Tm:Tn ratio of 7:2:2 with either skeletal or cardiac Tn. In all cases increasing the amount of Tm or Tn had no significant effect on the observed transients. A summary of the $k_{\text {obs }}$ values in the presence and absence of calcium and the derived $K_{B}$ values is shown in Table 2. These show that the ratio of the two rate constants (and hence $K_{B}$ ) is independent of the Tm isoform used. However, as noted previously, the ratio of the two rate constants is always smaller for cTn primarily because the value in the presence of calcium is smaller. Hence the value of $K_{\mathrm{B}}$ is $0.33 \pm 10 \%$ or $0.57 \pm 10 \%$ for skTn and cTn respectively for each of the Tm isoforms investigated.

Having an experimental system that defines a wellregulated thin filament we can then use the method of Head et al. (1995) to define the calcium sensitivity of the novel thin filaments. The result of one such assay is shown in Fig. 4. Figure 4A shows the fluorescence transient observed for the binding of $0.25 \mu \mathrm{M} \mathrm{S} 1$ to a ten-fold excess of actin saturated with rabbit-skeletal TmTn. The measurement was repeated for a range of $\mathrm{pCa}$ values between 4.6 and 9.0. As can be seen the transients are all well defined by a single exponential and the $k_{\text {obs }}$ value decreases as the calcium concentration is decreased. A plot of the $k_{\mathrm{obs}}$ against $\mathrm{pCa}$ is shown in Fig. 4B with the best fit to the Hill equation superimposed. This fit defined the mid-point of the calcium induced change in $k_{\mathrm{obs}}$ as pCa 6.05 with a Hill coefficient $\left(h_{C a}\right)$ of 1.47 .

A similar assay was performed for the $\alpha$-Tm and the $\beta$-Tm with both skeletal and cardiac Tn. The pCa plots are shown in Fig. 4B and the best-fit parameters are listed in Table 1. In the presence of skTn the results for the two homodimers of $\mathrm{Tm}$ are indistinguishable with a mid-point of the pCa curve occurring at $\mathrm{pCa} 6.05$ and a Hill coefficient of 1.5-1.68. In the presence of cTn the Hill coefficient for both Tms is $1.15-1.20$, smaller than for skTn as expected as $\mathrm{cTnC}$ has single calcium binding site. The mid point $\mathrm{pCa}$ is lower for both Tms again as expected for $\mathrm{cTn}$ compared to skTn but now the two Tms differ $\mathrm{pCa} 5.80$ for $\beta$-Tm and 5.57 for $\alpha$-Tm. Comparing the data for the expressed homodimers with the native Tm shows no significant differences

The $\mathrm{pCa}$ measurement was repeated using the $\alpha$-Tm carrying either of the two FHC mutations, D175N or E180G again using both sk and $\mathrm{cTn}$. The pCa plots are shown in Fig. $4 \mathrm{C}$ together with a replot of the $\alpha$-Tm from Fig. 4B for ease of comparison. The best-fit parameters to the Hill equation are given in Table 1. For cTn the Hill coefficients were unchanged for the $3 \alpha \mathrm{Tms}$ while the midpoints increase for the two mutants by $0.09 \mathrm{pCa}$ for $\mathrm{D} 175 \mathrm{~N}$ and $0.13 \mathrm{pCa}$ for E180G. Using skTn, the mid point of the $\mathrm{pCa}$ curves showed smaller changes for the mutants than with cTn (0.03 for D175N, 0.10 for E180G) but a more pronounced decrease in the Hill coefficient was observed from 1.50 to 1.22 for $\mathrm{D} 175 \mathrm{~N}$ and to 1.19 for E180G. The significance of these results will be considered in the Discussion. 
Table $2 K_{\mathrm{B}}$ measurements of $\mathrm{S} 1$ binding to regulated actin filaments assembled from different $\mathrm{Tm}$ and $\mathrm{Tn}$ isoforms

\begin{tabular}{|c|c|c|c|c|c|c|}
\hline & \multicolumn{4}{|l|}{$k_{\mathrm{obs}}$} & \multirow{2}{*}{\multicolumn{2}{|c|}{$K_{\mathrm{B}}$}} \\
\hline & \multicolumn{2}{|l|}{ skTn } & \multicolumn{2}{|l|}{$\mathrm{cTn}$} & & \\
\hline & $+\mathrm{Ca}^{2+}$ & $-\mathrm{Ca}^{2+}$ & $+\mathrm{Ca}^{2+}$ & $-\mathrm{Ca}^{2+}$ & skTn & cTn \\
\hline Native $\alpha \operatorname{Tm}$ & 4.50 & 1.20 & 3.40 & 1.25 & 0.35 & 0.55 \\
\hline$\alpha \mathrm{Tm}$ & 4.35 & 1.15 & 3.20 & 1.25 & 0.35 & 0.66 \\
\hline$\beta \mathrm{Tm}$ & 4.55 & 1.10 & 3.35 & 1.15 & 0.33 & 0.55 \\
\hline $\operatorname{SkTm}(\alpha \alpha / \alpha \mathrm{B})$ & 4.50 & 1.35 & 2.85 & 1.10 & 0.42 & 0.60 \\
\hline D175N. $\alpha-T m$ & 4.50 & 1.10 & 3.20 & 1.15 & 0.32 & 0.56 \\
\hline E180G. $\alpha-\mathrm{Tm}$ & 4.40 & 1.10 & 3.40 & 1.30 & 0.35 & 0.61 \\
\hline
\end{tabular}

$k_{\mathrm{obs}}$ - the observed exponential rate constant for S1 binding to excess thin filaments at $2.5 \mu \mathrm{M}$ actin. The value of $k_{\mathrm{obs}}$ in the absence of Tn was $5.30 \pm 0.2$ in each case. The $K_{\mathrm{B}}$ value (the equilibrium between blocked and closed states of the thin filament) was calculated using the equation $k_{\text {obs }}\left(-\mathrm{Ca}^{2+}\right) / k_{\text {obs }}\left(+\mathrm{Ca}^{2+}\right)=K_{\mathrm{B}} /\left(1+K_{\mathrm{B}}\right)$. The experimental conditions were as described in Fig. 3 legend. In the presence of tissue purified control protein complex (skTm.Tn complex) the $k_{\mathrm{obs}}$ values obtained were $4.8 \mathrm{~s}^{-1}$ and $1.5 \mathrm{~s}^{-1}$ for (+) and (-) $\mathrm{Ca}^{2+}$ respectively

\section{Discussion}

In the work presented here we were interested in two major issues. Firstly, the extent to which the fast kinetic analysis that we have developed can be used to define the calcium sensitivity of a wide range of muscle thin filament proteins. Secondly, the role of the isoforms of Tm and Tn and the interaction between them in defining the calcium sensitivity of the thin filament.

The first requirement of any quantitative assay of a complex system like the muscle thin filament, which is assembled from five different protein chains, is to have a simple convenient assay that can establish that a correctly assembled filament has been achieved. This is not a trivial matter as less than $100 \%$ activity of any one of the components can severely limit the validity or at least the reproducibility of the assays. The relatively simple assay of Fig. 3 provides several criteria to establish a good thin filament preparation; the stoichiometry of the actin:Tm:Tn assembled, the degree to which Tn in the absence of calcium can turn the filament off (compared to a pure actin or actin.Tm filament) and the degree to which calcium can restore the full activity of the filament. In our hands actin and Tm always bind to each other at very close to the correct 7:1 ratio whether tissue purified or recombinant Tm is used. Tissue purified rabbit skeletal $\mathrm{Tn}$ is very reliable and assembles in a $\mathrm{A}_{7} \mathrm{Tm}$ Tn complex, which inhibits the rate of $\mathrm{S} 1$ binding by no more than $10-15 \%$ in the presence of calcium and by a further 3.5-4 fold when calcium is removed. Bovine cardiac Tn (tissue purified or recombinant, (Reiffert et al. 1996, 1999), or recombinant, human-cardiac Tn (Lohmann et al. 2001) behave differently. The cTn assembles more reliably when TnI is phosphorylated by PKA than when dephosphorylated. Only fully phosphorylated Tn was used in the experiments reported here. The filament assembles with an actin:Tn ratio of between 7:1 and 7:2 to give a maximally inhibited $\mathrm{S} 1$ binding and the maximal inhibition is similar to that observed with skTn. The difference is that addition of calcium does not restore the rate of S1 binding to the same extent as skTn, the rate in the presence of calcium being typically 25-30\% lower than that for pure actin. This inhibition in the presence of calcium could be due to one of three things: 1, the presence of some damaged Tn component, which is inhibitory but does not activate on adding calcium. 2, That correctly assembled cTn does not activate fully on adding calcium (some blocked state always remains present); or 3 , That cTn has an indirect effect on $\mathrm{S} 1$ binding by reducing $k$, the second order rate constant for $\mathrm{S} 1$ binding. At present the observed result appears consistent over a number of preparations over several years and we suspect therefore that it may be an intrinsic property of cTn implying that either some blocked state does remain in the presence of calcium or that $k$ is altered. Equilibrium binding data using cardiac Tn in the presence of calcium does indicate that the filament is fully switched on and the data is indistinguishable from equivalent data for skTn (Maytum et al. 2000). There is unlikely therefore, to be any significant blocked state present in the presence of calcium and we assume this affect is due to an altered second order rate constant for S1 binding to actin.Tm.cTn.

The data of Table 1 establishes that recombinant Tm carrying the AS N-terminal extension is a good analogue of the native Tm, at least for the $\alpha \alpha$ isoform. The affinity of AS. $\alpha \alpha-$ Tm and AS. $\beta \beta$-Tm for actin is tight $(\sim 0.2 \mu \mathrm{M})$, quite similar and both bind with a 7:1 stoichiometry and assemble reliably with both skTn and cTn. The only marginal difference between the two was in the Hill coefficient $\left(h_{A}\right)$ for binding of Tm to actin 2.7 for $\beta \beta$ compared to 1.95 for $\alpha \alpha$-Tm. Looking at the data in Fig. 2 this difference does not appear to be very well defined and is unlikely to be of physiological relevance. A comparison to the $\alpha \beta$-Tm 

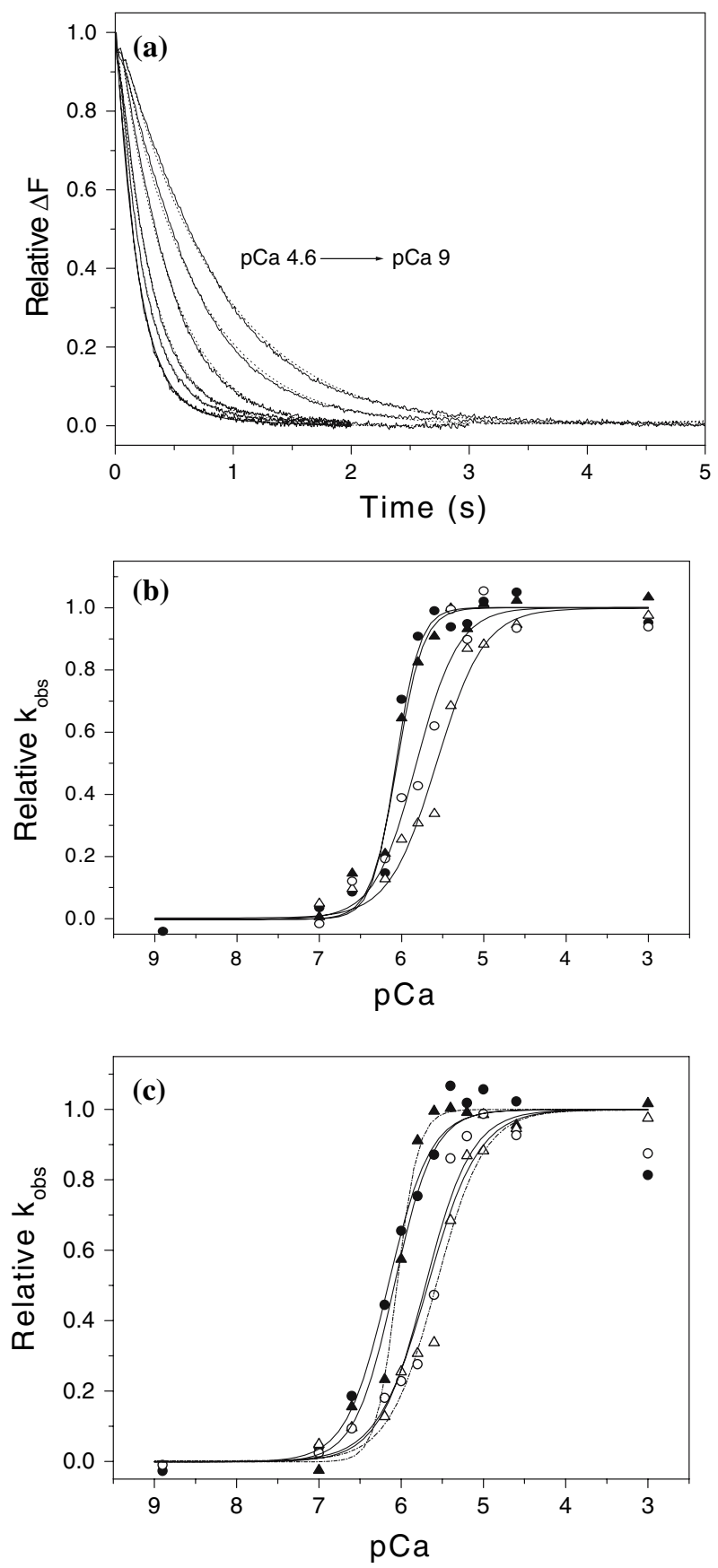

heterodimer is required and we are currently refining the method for assembly of the heterodimer.

All actin.Tm filaments switch on more readily (a higher pCa for the mid-point and larger $h_{C a}$ ) with skTn than with cTn. Calcium binding to TnC is different for cardiac versus skeletal Tn partly because of the different number of calcium binding sites in the regulatory domain of $\mathrm{TnC}$. However the calcium affinity is also influenced by the interaction of $\mathrm{TnC}$ with the other $\mathrm{Tn}$ components and their interaction with Tm and actin (for review see Gordon et al. 2001). Our assays detect these small differences and this
4Fig. 4 Dependence of the rate of S1 binding to pyrene-actin.Tm.Tn on calcium concentrations and on the isoforms of Tm and Tn. (A) Observed transients for excess pyrene actin $(2.5 \mu \mathrm{M}$ and $1 \mu \mathrm{M}$ skTm.Tn) binding to $0.25 \mu \mathrm{M}$ S1.The best fit single exponentials (dotted line) are superimposed and gave $k_{\mathrm{obs}}$ values of : $5.2 \mathrm{~s}^{-1}$ (pCa 4.6), $4.25 \mathrm{~s}^{-1}$ (pCa 5.6), 3.4 s $\mathrm{s}^{-1}$ (pCa 6), $2.2 \mathrm{~s}^{-1}$ (pCa 6.2), $1.55 \mathrm{~s}^{-1}$ (pCa 6.6) and $1.2 \mathrm{~s}^{-1}$ (pCa 9). (B) Observed $\mathrm{pCa}$ curves in the presence of AS. $\alpha-\operatorname{Tm}(\Delta)$ or AS. $\beta$-Tm $(O)$ and either cardiac (open symbols) or skeletal (filled symbols) Tn. A best fit to the Hill equation is superimposed with $\mathrm{pCa}$ midpoints and Hill coefficients shown in Table 1. (C) Observed pCa curves in the presence of AS. $\alpha-\mathrm{Tm}$ mutants D175N $(O)$ and E180G $(\Delta)$ associated with either cardiac (empty symbols) or skeletal (filled symbols) Tn. pCa midpoint values and Hill coefficients are listed in Table 1. The fitted curve of the WT $\alpha$-Tm data from B is shown by a dotted line for ease of comparison. Experimental conditions: as for Fig. 3. Plus a combination of $2 \mathrm{mM}$ EGTA and $2 \mathrm{mM}$ Ca-EGTA to produce the defined pCa values (see methods). cTn was phosphorylated by PKA

demonstrates the potential of this approach for exploring the information pathways through the actinTmTn complex. We have previously shown that the method can also detect the shift in the mid-point of the pCa curve caused by phosphorylation of TnI in cTn (Reiffert et al. 1996).

A comparison of thin filaments containing $\alpha \alpha$ vs. $\beta \beta$-Tm shows some interesting differences particularly in the presence of $\mathrm{cTn}$. The $\beta \beta$-Tm with $\mathrm{cTn}$ is more calcium sensitive by $0.23 \mathrm{pCa}$ units $(1.10 \mu \mathrm{M}$ shift in calcium concentration) compared to $\alpha \alpha$-Tm with no measurable difference in the Hill coefficient. In contrast in the presence of skTn the calcium sensitivity was indistinguishable with a slightly larger value of the Hill coefficient for the $\beta$ isoform but the difference is small (1.50 vs. 1.68). The data presented here are consistent with data obtained by over expression of $\beta$-Tm in mouse heart, which also showed an increase in calcium sensitivity (Palmiter et al. 1996).

A detailed interpretation of the sequence differences between $\alpha \& \beta \mathrm{Tm}$ is beyond the scope of the current work but the comparison shown in Fig. 1 reveals some features of interest. The coiled-coil structure of Tm is defined by the heptad repeat (abcdefg) of the amino acid sequence in which positions $a \& d$ are small hydrophobic residues and form the primary interaction between the two chains, positions $e \& g$ can provide secondary contacts that are usually ionic in character. Of the 12 non-conservative sequence changes, three are at positions to directly influence coiled-coil stability (one at position $a$ of the heptad repeat and two at postioins $g / e$ ). There are a further two Ala-toSer substitutions and one Ser-to-Ala substitutions at $d$ positions of the heptad which are of interest in relation to the suggestion that alanine clusters at the coiled-coil interface are involved in defining the flexibility of Tm (Brown et al. 2001). The remaining 9 non-conservative subsitutions are at are at the heptad positions $b, c, \& f$, so they are unlikely to influence the coiled-coil stability directly but can alter the interaction of Tm with its binding 
partners. $\mathrm{Tm}-\mathrm{Tm}$ interactions are thought to occur in the overlap between the 8-10 N \& C-terminal amino acids (Greenfield et al. 2001). There are no sequence changes at the $\mathrm{N}$-terminus but three conservative changes at the C-terminus. This overlap region is also thought to be a site of interaction of the N-terminal part of TnT. The major Tn interaction site is about one third of the length of Tm away from the C-terminus and close to the alternatively spliced exon 6 (see Fig. 1). In this region there are four non-conservative sequence changes (G188S, A191G, T199I, $\mathrm{Q} 216 \mathrm{~T})$ and two conserved E to D changes. These are therefore in a position to alter the interaction of Tn with $\alpha \alpha$ and $\beta \beta \mathrm{Tm}$ as shown by the effects of exon 6 substitutions on calcium sensitivity (Maytum et al. 2004).

The AS. $\alpha \alpha$-Tm carrying one of the two FHC mutations have been studied by several groups (Golitsina et al. 1997; Bottinelli et al. 1998; Bing et al. 2000; Chang et al. 2005). We previously reported that there was a small difference in the affinities of the two proteins for actin compared to $\alpha \alpha$ and that the E180G mutation was less thermally stable than WT protein both in the presence and absence of actin (Kremneva et al. 2004). Here we show that D175N Tm shows little change in calcium sensitivity compared to WT using either skTn $(+0.03 \mathrm{pCa})$ or cTn $(+0.09 \mathrm{pCa})$. E180G shows a slightly larger shift $(+0.10$ and +0.13 respectively). The data for D175N are within the error of the measurement while that for E180G is about twice the standard error. This is clearly seen in the data plotted in Fig. 4B as is the smaller cooperativity for E180G filaments with skTn but not cTn. These differences are at the limit of the sensitivity of the assay. A $0.1 \mathrm{pCa}$ unit shift of calcium sensitivity could be sufficient to cause a problem in the heart (phosphorylation of TnI is used to generate only 0.2 $-0.3 \mathrm{pCa}$ unit shift). The precision of the measurements would need to be improved to be confident that such a change is attributable to the mutation. The possibility remains that for E180G at least, the loss of stability near body temperature could cause a change in calcium sensitivity at temperatures close to $37^{\circ} \mathrm{C}$.

The conclusion from these studies is that the rapid kinetic assay can provide a convenient tool for estimating the calcium sensitivity of thin filaments assembled from different combinations of $\mathrm{Tm}$ and $\mathrm{Tn}$ isoforms. The assays provide a direct measure of the fraction of actin sites unavailable to bind $\mathrm{S} 1$ and the calcium sensitivity and cooperativity of the switch between off and on states. We have shown differences between $\alpha \alpha, \beta \beta$-Tm and $\alpha \alpha-\mathrm{Tm}$ carrying point mutations linked to hypertrophic cardiomyopathy. The differences observed depend upon which Tn isoform is used, cardiac or skeletal. The differences observed illustrate the subtle interaction between the components of the thin filament and indicate why single point mutations in $\mathrm{Tm}$, for example, can lead to life-threatening myopathies in the heart yet the same mutation produces no apparent phenotype in skeletal muscle. Future studies will need to address the differences between homodimers and heterodimers of $\mathrm{Tm}$, which will include $\alpha \beta$-Tm and Tm in which only one monomer carries a mutation.

The small values of the Hill coefficients for the calcium switching of the thin filaments provided little evidence to support long-range cooperativity along the filaments. The Hill coefficient of $\sim 1.5$ for skTn is consistent with 2 calcium ions being required to switch on each $\mathrm{Tn}$ with little Tn-Tn cooperativity. For the cardiac Tn the Hill coefficient is above 1 , consistent with some $\mathrm{Tn}-\mathrm{Tn}$ cooperativity, but not by a very large margin.

Acknowledgements This work was supported by a British Heart Foundation Studentship to SEB (FS/2000014) and by the NIH (AR048776) and the Wellcome Trust (Program Grant No 070021). We thank Darl Swartz and Sherwin Lehrer for helpful comments on the manuscript and $\mathrm{N}$ Adamek for technical support.

\section{References}

Bing W, Knott A et al (2000) Effect of hypertrophic cardiomyopathy mutations in human cardiac muscle alpha -tropomyosin (Asp175Asn and Glu180Gly) on the regulatory properties of human cardiac troponin determined by in vitro motility assay. J Mol Cell Cardiol 32:1489-1498

Bottinelli R, Coviello DA et al (1998) A mutant tropomyosin that causes hypertrophic cardiomyopathy is expressed in vivo and associated with an increased calcium sensitivity. Circ Res 82:106-115

Brown JH, Kim KH et al (2001) Deciphering the design of the tropomyosin molecule. Proc Natl Acad Sci USA 98:8496-8501

Chang AN, Harada K, Ackerman MJ, Potter JD (2005) Functional consequences of hypertrophic and dilated cardiomyopathycausing mutations in alpha-tropomyosin. J Biol Chem 280:34343-34349

Chen Y, Yan B, Chalovich JM, Brenner B (2001) Theoretical kinetic studies of models for binding myosin subfragment-1 to regulated actin: Hill model versus Geeves model. Biophys J 80:2338-2349

Craig R, Lehman W (2001) Crossbridge and tropomyosin positions observed in native, interacting thick and thin filaments. J Mol Biol 311:1027-1036

Criddle AH, Geeves MA, Jeffries T (1985) The use of actin labelled with $\mathrm{N}$-(1-pyrenyl)iodoacetamide to study the interaction of actin with myosin subfragments and troponin/tropomyosin. Biochem J 232:343-349

Deng Y, Schmidtmann A et al (2001) Effects of phosphorylation and mutation R145G on human cardiac troponin I function. Biochemistry 40:14593-14602

Ebashi S, Wakabayashi T, Ebashi F (1971) Troponin and its components. J Biochem (Tokyo) 69:441-445

Golitsina N, An Y et al (1997) Effects of two familial hypertrophic cardiomyopathy-causing mutations on alpha-tropomyosin structure and function. Biochemistry 36:4637-4642

Gordon AM, Homsher E, Regnier M (2000) Regulation of contraction in striated muscle. Physiol Rev 80:853-924

Gordon AM, Regnier M, Homsher E (2001) Skeletal and cardiac muscle contractile activation: tropomyosin "rocks and rolls". News Physiol Sci 16:49-55 
Greaser ML, Gergely J (1971) Reconstitution of troponin activity from three protein components. J Biol Chem 246:4226-4233

Greenfield NJ, Huang YJ et al (2001) Solution NMR structure and folding dynamics of the $\mathrm{N}$ terminus of a rat non-muscle alphatropomyosin in an engineered chimeric protein. J Mol Biol 312:833-847

Harrison SM, Bers DM (1987) The effect of temperature and ionic strength on the apparent Ca-affinity of EGTA and the analogous Ca-chelators BAPTA and dibromo-BAPTA. Biochim Biophys Acta 925:133-143

Head JG, Ritchie MD, Geeves MA (1995) Characterization of the equilibrium between blocked and closed states of muscle thin filaments. Eur J Biochem 227:694-699

Heeley DH, Belknap B, and White HD (2006) Maximal activation of skeletal muscle thin filaments requires both rigor myosin $\mathrm{S} 1$ and calcium. J Biol Chem 281:668-676

Heeley DH, Dhoot GK et al (1983) The effect of cross-innervation on the tropomyosin composition of rabbit skeletal muscle. FEBS Lett 152:282-286

Helfman DM (1994) The generation of protein isoform diversity by alternative RNA splicing. Soc Gen Physiol Ser 49:105-115

Jagatheesan G, Rajan S et al (2003) Functional importance of the carboxyl-terminal region of striated muscle tropomyosin. J Biol Chem 278:23204-23211

Kremneva E, Boussouf S et al (2004) Effects of two familial hypertrophic cardiomyopathy mutations in alpha-tropomyosin, Asp175Asn and Glu180Gly, on the thermal unfolding of actinbound tropomyosin. Biophys J 87:3922-3933

Laemmli UK (1970) Cleavage of structural proteins during the assembly of the head of bacteriophage T4. Nature 227:680-685

Lees-Miller JP, Helfman DM (1991) The molecular basis for tropomyosin isoform diversity. Bioessays 13:429-437

Lehrer SS, Joseph D (1987) Differences in local conformation around cysteine residues in alpha alpha, alpha beta, and beta beta rabbit skeletal tropomyosin. Arch Biochem Biophys 256:1-9

Lehrer SS, Qian Y (1990) Unfolding/refolding studies of smooth muscle tropomyosin. Evidence for a chain exchange mechanism in the preferential assembly of the native heterodimer. J Biol Chem 265:1134-1138

Lehrer SS, Qian YD, Hvidt S (1989) Assembly of the native heterodimer of Rana esculenta tropomyosin by chain exchange. Science 246:926-928

Lohmann K, Westerdorf B et al (2001) Overexpression of human cardiac troponin in Escherichia coli: its purification and characterization. Protein Expr Purif 21:49-59

Maytum R, Bathe F, Konrad M, Geeves MA (2004) Tropomyosin exon $6 \mathrm{~b}$ is troponin-specific and required for correct acto-myosin regulation. J Biol Chem 279:18203-18209

Maytum R, Geeves MA, Konrad M (2000) Actomyosin regulatory properties of yeast tropomyosin are dependent upon N-terminal modification. Biochemistry 39:11913-11920
Maytum R, Westerdorf B, Jaquet K, Geeves MA (2003) Differential regulation of the actomyosin interaction by skeletal and cardiac troponin isoforms. J Biol Chem 278:6696-6701

McKillop DF, Geeves MA (1993) Regulation of the interaction between actin and myosin subfragment 1: evidence for three states of the thin filament. Biophys J 65:693-701

Monteiro PB, Lataro RC, Ferro JA, Reinach FdeC (1994) Functional alpha-tropomyosin produced in Escherichia coli. A dipeptide extension can substitute the amino-terminal acetyl group. J Biol Chem 269:10461-10466

Muthuchamy M, Boivin GP, Grupp IL, Wieczorek DF (1998) Betatropomyosin overexpression induces severe cardiac abnormalities. J Mol Cell Cardiol 30:1545-1557

Muthuchamy M, Grupp IL et al (1995) Molecular and physiological effects of overexpressing striated muscle beta-tropomyosin in the adult murine heart. J Biol Chem 270:30593-30603

Palmiter KA, Kitada Y et al (1996) Exchange of beta- for alphatropomyosin in hearts of transgenic mice induces changes in thin filament response to $\mathrm{Ca} 2+$, strong cross-bridge binding, and protein phosphorylation. J Biol Chem 271:11611-11614

Perry SV (2001) Vertebrate tropomyosin: distribution, properties and function. J Muscle Res Cell Motil 22:5-49

Pirani A, Vinogradova MV et al (2006) An atomic model of the thin filament in the relaxed and $\mathrm{ca}(2+)$-activated States. J Mol Biol 357:707-717

Reiffert SU, Jaquet K et al (1996) Bisphosphorylation of cardiac troponin I modulates the $\mathrm{Ca}(2+)$-dependent binding of myosin subfragment $\mathrm{S} 1$ to reconstituted thin filaments. FEBS Lett 384:43-47

Reiffert S, Maytum R et al (1999) Characterization of the cardiac holotroponin complex reconstituted from native cardiac troponin $\mathrm{T}$ and recombinant I and C. Eur J Biochem 261:40-47

Schaertl S, Lehrer SS, Geeves MA (1995) Separation and characterization of the two functional regions of troponin involved in muscle thin filament regulation. Biochemistry 34:15890-15894

Spudich JA, Watt S (1971) The regulation of rabbit skeletal muscle contraction. I. Biochemical studies of the interaction of the tropomyosin-troponin complex with actin and the proteolytic fragments of myosin. J Biol Chem 246:4866-4871

Takeda S, Yamashita A, Maeda K, Maeda Y (2003) Structure of the core domain of human cardiac troponin in the $\mathrm{Ca}(2+)$-saturated form. Nature 424:35-41

Thierfelder L, Watkins H et al (1994) Alpha-tropomyosin and cardiac troponin $\mathrm{T}$ mutations cause familial hypertrophic cardiomyopathy: a disease of the sarcomere. Cell 77:701-712

Tobacman LS, Butters CA (2000) A new model of cooperative myosin-thin filament binding. J Biol Chem 275:27587-27593

Vinogradova MV, Stone DB et al (2005) Ca(2+)-regulated structural changes in troponin. Proc Natl Acad Sci USA 102:5038-5043

Weeds AG, Taylor RS (1975) Separation of subfragment-1 isoenzymes from rabbit skeletal muscle myosin. Nature 257:54-56 